\title{
A REVIEW OF GAMMA-RAY BURST OBSERVATIONS
}

\author{
W. Doyle Evans and John G. Laros \\ Los Alamos National Laboratory \\ Earth and Space Sciences Division \\ P.0. Box 1663 \\ Los Alamos, NM 87545
}

ABSTRACT. Gamma-ray bursts are generally believed to originate in the vicinity of neutron stars, but the phenomenology is still not understood. In this paper we review the known characteristics of gamma bursts and give new observational results on temporal and spectral properties. We suggest that a class of repeating bursters exists that are spectrally harder than $\mathrm{x}$-ray bursters but significantly softer than "classical" gamma bursts. The March 5, 1979 , burst may be the prototype of this class of bursters.

\section{INTRODUCTION}

Excellent reviews of the current observational situation in transient gamma-ray astronomy have been provided recently in three AIP Conference Proceedings Volumes. 1-3 These volumes also contain many details on the current research in this field. This paper will be a summary of these results in terms of temporal properties, spectral properties, and source locations. In addition, significant new results in each of the three areas will be discussed in more detail. To efficiently use the time available for the discussion of gamma bursts, we will limit our remarks to the observational data. Kevin Hurley, in the paper that follows, will then describe the inferences concerning neutron stars that can be drawn from the data.

\section{TEMPORAL PROPERTIES}

Gamma-ray bursts (GRBs) are characterized by short duration and extreme variability. Most bursts have a total duration of 1-10 s. Generally the time profiles consist of one or more intense peaks of varying widths separated by intervals of weak emission. Figure 1 is of historical interest and shows the type of time profiles first obtained by the Vela satellites. The instrumental characteristics prevented the acquisition of data during the initial rise and provided high-resolution data only during very early times. Figure 2 shows data from the Third International Sun-Earth Explorer (ISEE-3) instrument that provides a sampling period with pretrigger 


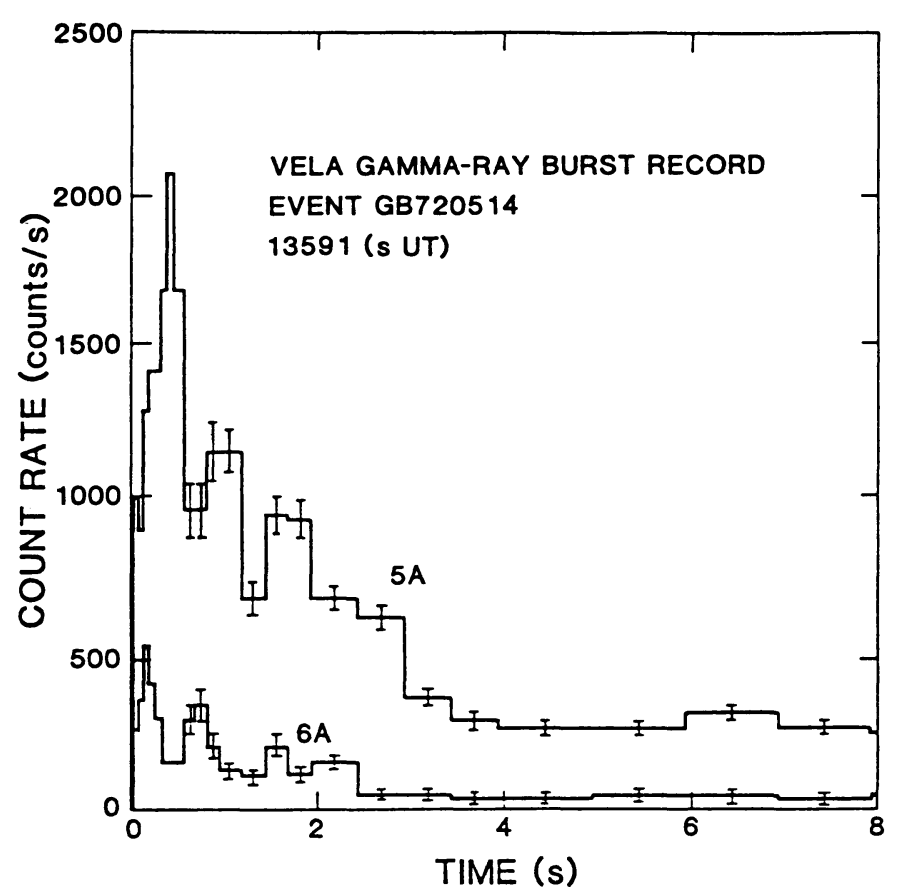

Figure 1. A gamma-ray burst time history recorded by detectors on the Vela $5 \mathrm{~A}$ and Vela $6 \mathrm{~A}$ satellites.
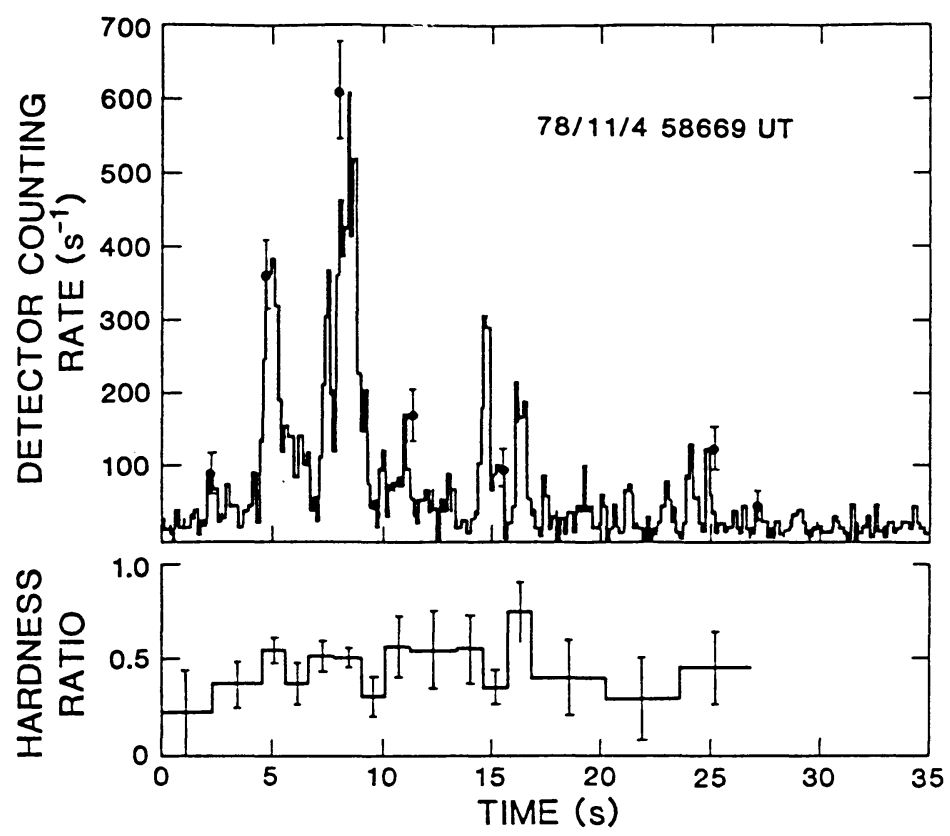

Figure 2. A gamma-ray burst time history recorded by the ISEE-3 detector. 
information and uniform time resolution throughout. The presence of well-defined peaks throughout the event is clearly shown.

There are notable exceptions to the typical 1-10-s duration, both shorter and longer. In particular several short events appear to consist of a single peak lasting for a few tenths of a second. A notable example of this type was the "March 5 Event," GB790305. Until recently it was the only short burst that was intense enough to allow detection of fine structure within the burst profile. Such fine structure was of low amplitude for this event--the burst had a very fast rise with a fairly smooth but irregular decay, followed by the well-known periodic afterglow. Recently Laros et al. 4 have published data from another very intense short burst that occurred on 1984 December 15. Unlike GB790305, GB841215 consisted of at least seven distinct, well-separated peaks with weak, nonperiodic emission following the main burst. This suggests that there could be at least two types of short bursts. It also suggests that we have not yet been able to observe the true characteristic time scale of the variability because of instrumental limitations. Since GB790305 and GB841215 were differentiated by their spectra as well as by their fine structure, we will return to this topic in the following section.

In another recent observation, Itoh et al. 5 have presented results obtained on 1984 March 4, from the Pioneer Venus Orbiter (PVO) instrument. As shown in Figure 3, this burst consisted of two

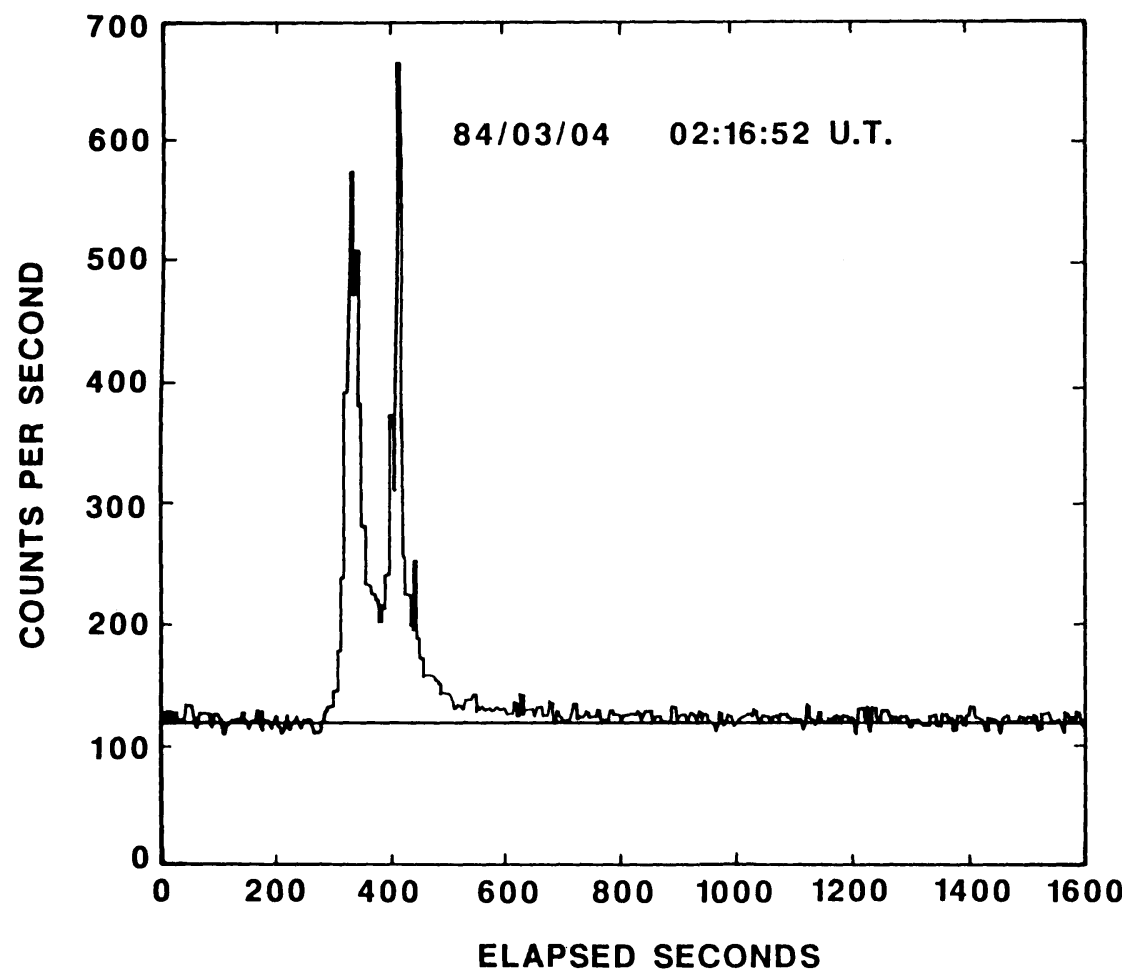

Figure 3. A gamma-ray burst recorded by the Pioneer Venus Orbiter. The two intense peaks are followed by more than $1000 \mathrm{~s}$ of spectrally hard emission. 
distinct peaks followed by over $1,000 \mathrm{~s}$ of weaker, but spectrally very hard, emission. Since GRB cooling times are believed to be extremely short, this observation implies that an energy source is active for at least $1000 \mathrm{~s}$. Burster models are severely constrained by this finding.

Searches for periodicities in gamma bursts have been largely unsuccessful; an obvious exception is the extended emission from GB790305. Also, Wood et al. 6 presented evidence for possible periodic structure in a burst that occurred on 1977 October 29. The 1979 January 13 event has been suggested by Barat et al.7 as having a possible periodicity. U. Desai at Goddard has proposed that several additional bursts show evidence for periodicities or quasi periodicities, but the evidence is not overwhelming. However, in a collection of several hundred gamma bursts, it might be expected that, simply due to chance, several will have peaks that are approximately evenly separated.

\section{SPECTRAL PROPERTIES}

As with other properties of gamma bursts, continued observation has revealed a rich diversity in the spectral shape of the emission and the spectral variability during the burst. Notwithstanding the wide differences between bursts, however, it is still true that, as a group, the spectra are distinctly different from any other astrophysical radiation source. Figure 4 from Epstein (private

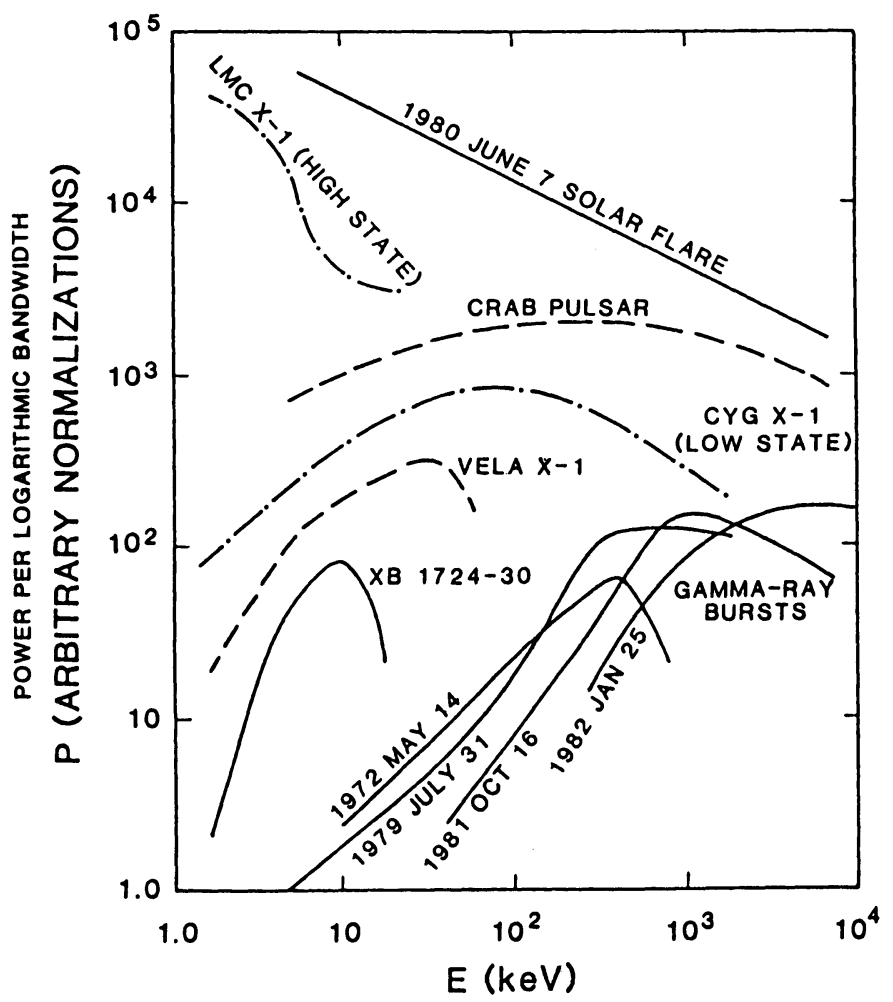

Figure 4. Typical spectra of various high-energy astrophysical sources. Gamma-ray bursts form a distinct class. 
communication) illustrates this difference clearly. Examples of the spectral shape of the emission from several types of transients are plotted in units of logarithmic intensity. Vertical normalization is arbitrary. The several gamma-burst spectra shown in the lower right are grossly different from $x$-ray bursts, $x$-ray transients, and solar flares. Most of the power in the bursts came from above $1 \mathrm{MeV}$. Matz ${ }^{8}$ has recently published excellent results from the Solar Maximum Mission showing a number of bursts with emission extending to several $\mathrm{MeV}$ without any apparent turnover in the continuum spectra. Figure 5 shows one such spectrum, best fit by a power law. Again, instrumentation limits our knowledge at the highest energies.

In the $x$-ray region of the spectrum the observations are limited, but sufficient data exist to support some important conclusions.9,10 Very little of the power is emitted below $30 \mathrm{keV}$-approximately $1 \%$. The soft emission has a very different time profile than does the harder portion of the spectrum; i.e., the overall spectral shape varies widely during a burst, even though the shape above $100 \mathrm{keV}$ remains relatively unchanged. Some investigators have suggested that the observations are most easily understood as two distinct components-soft and hard--that are not strongly coupled at the source.

Golenetskii et al.11 have suggested a correlation between intensity and spectral shape and have published several examples illustrating this correlation. Other investigators have not been able to confirm this relationship and have instead suggested that the spectra are hardest on the leading edges of the individual peaks. 12,13 GB840304 has been analyzed and the interesting result is shown in Figure 6 . These data are for the two peaks shown in Figure 3. Each point is

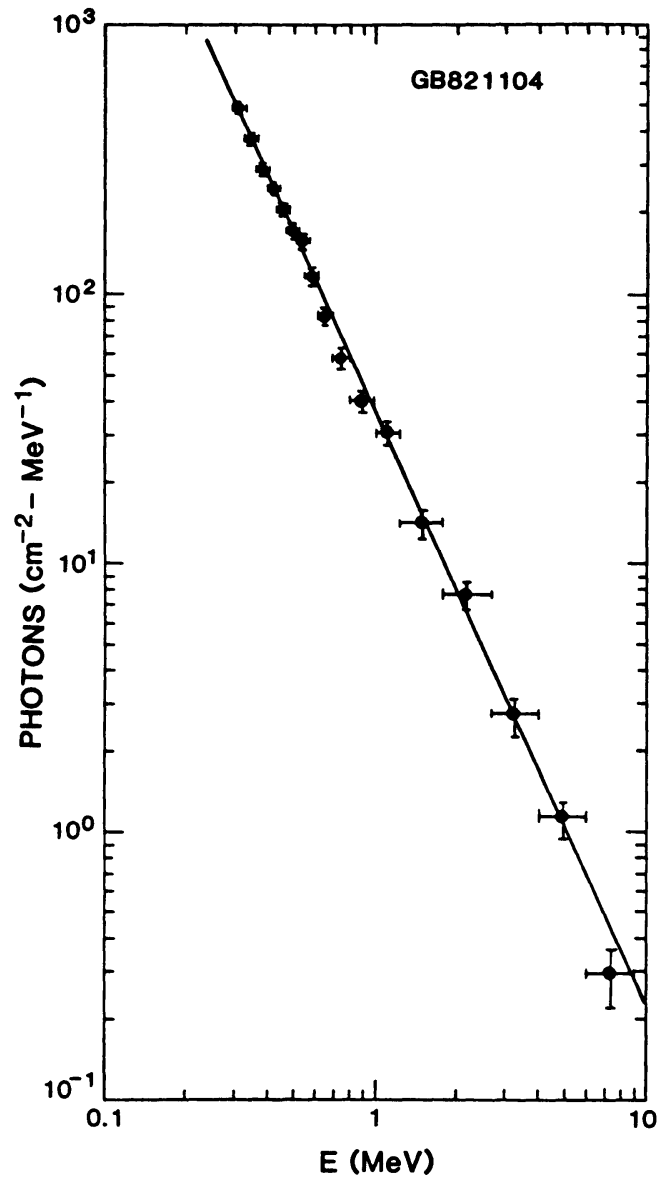

Figure 5. Gamma-ray burst spectrum obtained from the Solar Maximum Mission (Matz, ref. 8) showing emission extending to 10 $\mathrm{MeV}$. 


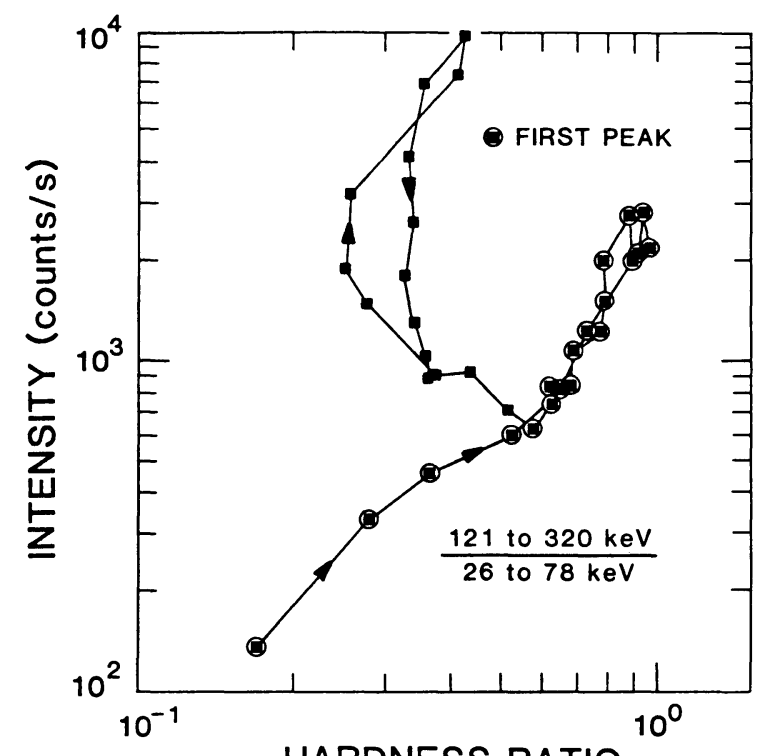

Figure 6. Hardness ratio vs. intensity for the two peaks of the GB840304 (See Figure 3). During the first peak (circled squares) there is a strong correlation. This correlation completely disappears during the second peak (solid squares).

derived from $4.5 \mathrm{~s}$ of data, and the arrows show the direction of time. During the early portion of this peak, there is a strong positive correlation between intensity and spectral hardness. However, this relationship breaks down completely after approximately $90 \mathrm{~s}$, and the intensity becomes roughly spectrum independent. Apparently there are bursts, or at least portions of bursts, for which the intensityspectrum correlation holds, but as is usual for gamma bursts, there is no consistent behavior. Certainly, it has been shown that GRB spectra can vary as rapidly as their overall time histories and that future spectral analyses must deal with this fact.

The presence (or absence) of spectral features has been the subject of considerable debate in the past. Several spectra published by Mazets et al.,14 show significant departures from a smooth continuum in the energy range between 400 and $500 \mathrm{keV}$. The presence of gravitationally shifted annihilation radiation could produce the observed spectral features, and this was interpreted as strong evidence that the emission originated at the surfaces of neutron stars. Also, apparent absorption features below $100 \mathrm{keV}$ were explained as synchrotron absorption in magnetic fields of several $\times 10^{12} \mathrm{G}$--again consistent with magnetized neutron stars. However, this interpretation was questioned by Fenimore et al.,15 who argued that an incorrect assumption concerning the continuum shape coupled with 
instrumental effects could produce the observed results, and by Epstein (private communication), who pointed out that $>10^{12}-$ G magnetic fields would significantly deplete the continuum emission at high energies. Alternatively, the features could be caused by the superposition of two or more different emission components. 10 Confirmation of the features by other observational groups has been inconsistent. Certainly at this time even the reality of the spectral features is not completely established.

Spectral measurements have helped provide evidence that a few of the catalogued GRBs might belong to a separate class of gamma-ray transients. It has already been noted that GB790305 had, in addition to its other remarkable properties, an unusually soft spectrum for a GRB. Subsequently, it was discovered that GB790305 and another soft burster, GB790324, were repetitive.16 (Normal, hard-spectrum GRBs have never been observed to repeat.) Based on its soft spectrum and short duration, GB790107 was pointed out as a possibly similar object.17,18 Very recently we discovered perhaps more than 50 repetitions of GB790107 that occurred in late 1983. Thus, it appears likely that a new class of short, rapidly repeating, soft gamma-ray transients exists.

\section{SOURCE LOCATIONS}

Although the directions to $\sim 100$ GRBs have been determined, 16,19 the ir locations in space (i.e., their distances) remain unknown. Since the directions do not seem to favor either the Galactic center or the Galactic plane, the sources must be either very nearby or very distant, compared with the diameter of the Galaxy. Attempts to resolve this ambiguity through analysis of the $\log N-\log S$ or $\log N-\log P$ data have been inconclusive. Jennings 20 has devoted considerable effort to this study and concludes that an extended Galactic halo best explains the observations. Conversely, Higdon and Lingenfelter 21 claim that the departure of $\log \mathrm{N}-\log \mathrm{g}$ from the $3 / 2$ power-law slope is totally the result of selection effects produced by instrumental design and variations. The most recent results from Klebesadel (private communication) using PVO data show no significant departure of the $\log N-\log P$ curve from the $3 / 2$ slope (Figure 7 ), except that due to the instrumental threshold. These results, all from the same instrument and all based on peak intensity rather than total fluence, are compatible with either distance scale. Apparently an increase in instrumentation sensitivity of at least an order of magnitude will be

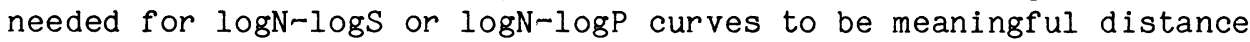
indicators.

GRB observers have attempted to follow the example of $x$-ray astronomy, where association of $x$-ray sources with optically identifiable astronomical systems has produced tremendous progress. Interplanetary networks, in a commendable example of international cooperation, have provided several very precise ( arcmin) error boxes through time-of-arrival analysis techniques. 19 with only a few notable exceptions, these error boxes have contained no interesting 


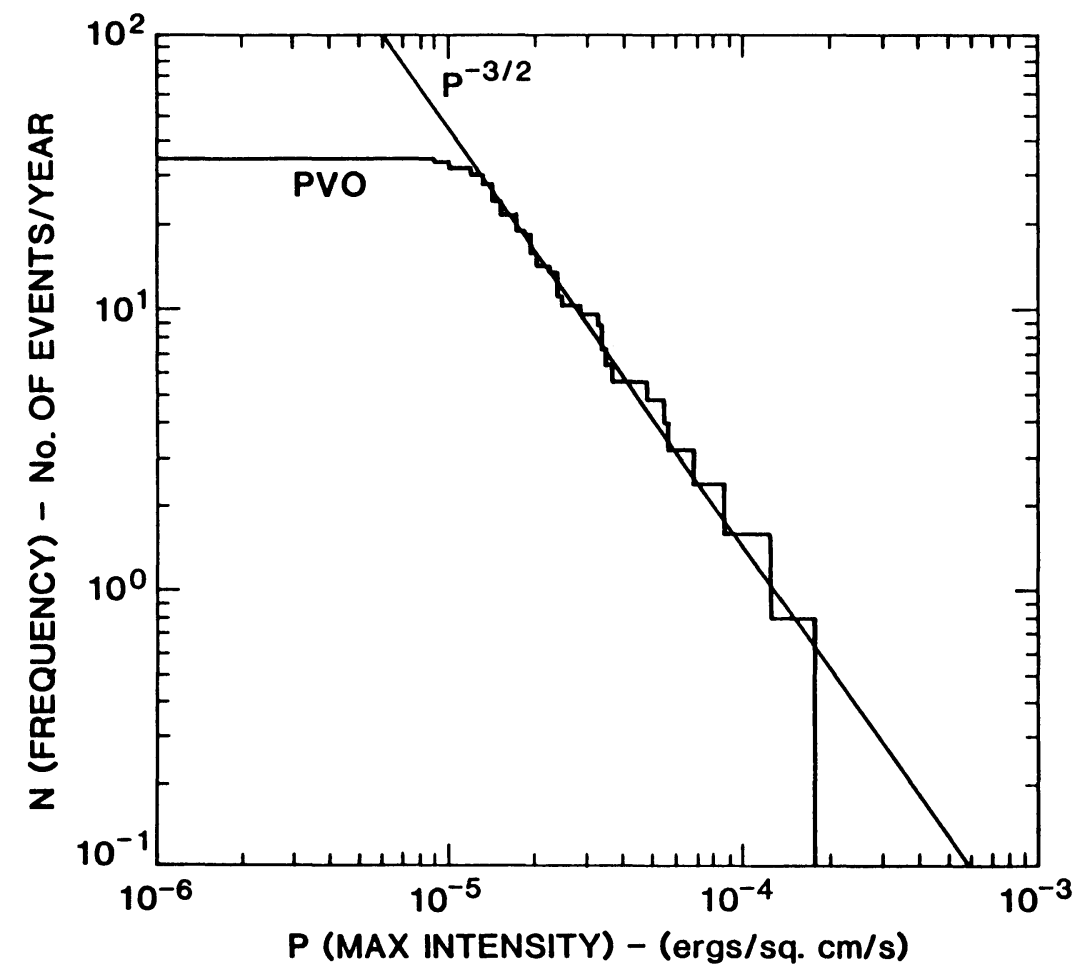

Figure 7. LogN vs. LogS data for all Pioneer Venus Orbiter data. The 35 events are consistent with a $-3 / 2$ law.

objects or no observable objects at all. The first exception was the March 5 event, whose direction was found to be coincident with the LMC supernova remnant $\mathrm{N} 49$. More recently, Schaefer 22 has discovered probable optical flashes on archival photographs of three GRB error boxes. However, the LMC association has been questioned on energetics grounds, and the optical flashes have not led to the firm identification of any quiescent counterpart. In any case, we have learned that gamma-ray bursters in quiescence are extremely faint objects.

\section{CONCLUSIONS}

New temporal observations have shown that even the shortest class of gamma burst, previously observed only as single peaks, can be composed of many faster, distinct peaks that are observable when there is sufficient count rate. Even better observations may be required to determine the characteristic time scale of the variability. Also, the extended, hard emission from GB840304 seems to require an energy source that remains available for over $1000 \mathrm{~s}$, thus adding a major constraint to virtually all gamma-burst models.

New time-resolved spectral measurements indicate that interpretations. of spectral data have been seriously limited by the fact that the burst spectra evolve on time scales at least as short as the 
fastest accumulation times of the present spectroscopic instruments. Time-averaged spectra may contain artifacts produced by this variability and must therefore be treated with caution. Even so, the high-energy spectral data from the Solar Maximum Mission are important in that they constrain important parameters of the emitting region, particularly the magnetic field. Correlations between intensity and spectral hardness are clearly present during some portions of some bursts, but they are not always present and their origins are unknown. Spectral measurements have also led to the identification of a possible new class of short, rapidly repeating, soft gamma-ray transient.

The new GRB location information probably has been less illuminating. Attempts to use the $\sim 100$ approximately known directions, together with $\operatorname{logN}-\log S$ or $\log N-\log P$ curves to establish a distance scale, have been inconclusive. Furthermore, the several available precise directions have not led to the identification of a gamma-ray burst counterpart.

\section{$\underline{\text { References }}$}

1. R. E. Lingenfelter, H. S. Hudson, and D. M. Worrall, eds., AIP Conf. Proc. No. 77, La Jolla (1982).

2. S. E. Woosley, ed., AIP Conf. Proc. No. 115, Santa Cruz (1984).

3. E. P. Liang and V. Petrosian, eds., AIP Conf. Proc. No. 141, Stanford (1986).

4. J. G. Laros et al., Nature 318, 448 (1985).

5. M. Itoh et al., Bull. Am. Astron. Soc. 17, 850 (1985).

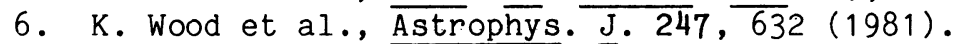

7. C. Barat et al., Astrophys. J. (Lett.) 286, L5 (1984).

8. S. M. Matz, 'On the Spectra of Gamma-Ray Bursts at High Energies,' Ph.D. dissertation, U. of New Hampshire (1986).

9. J. G. Laros et al., Astrophys. J. 286, 681 (1984).

10. J. G. Laros and J. Nishimura, A $\bar{I} P$ Conf. Proc. No. 141, 79 (1986).

11. S. Golenetskii et al., Nature 307, 41 (1984).

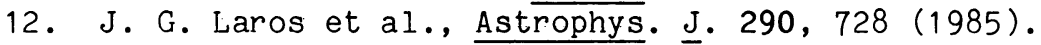

13. J. P. Norris et al., Bull. Am. Astron. Soc. 16, 447 (1984).

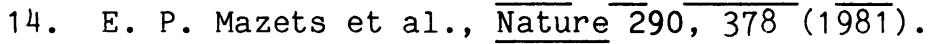

15. E. E. Fenimore et al., AIP Conf. Proc. No. 77, 201 (1982).

16. E. P. Mazets et al., Astrophys. Space sci. $\overline{80}, 3$ (1981).

17. E. P. Mazets et al., Astrophys. Space Sci. 84, 173 (1982).

18. J. G. Laros et al., Nature 322, $\overline{152(1986)}$.

19. J. -L. Atteia et al., Astrophys. J. (Supp.), in press (1986).

20. M. Jennings, AIP Conf. Proc. No. $115, \overline{412}$ (1984).

21. J. Higdon and $\mathrm{R}$. Lingenfelter, $A I \bar{P}$ Conf. Proc. No. 115, 568 (1984).

22. B. E. Schaefer, AIP Conf. Proc. No. 115, 406 (1984). 


\section{DISCUSSION}

E. Liang: Have you looked at the $\log N-\log S$ distributions of the $V^{2}{ }^{2}$ 's as a class? Are all of them much weaker than the March 5 th event.

D. Evans: Not yet. But they are weaker than March 5, 1979.

S. Woosley: With the discovery of $\mathrm{VS}^{2} \mathrm{Bs}$ the void in energy space you referred to in the beginning of your talk separating X-ray bursts and $\gamma$-ray bursts appears to be filled in, suggesting if not a continuity of mechanism, at least that nature has been resourceful in providing transients over a broad, continuous range of energies, i.e., a large fraction of so called " $\gamma$-ray bursts" emit most of their energy at "hard X-ray" wavelengths.

D. Evans: The VS ${ }^{2}$ Bs certainly fall between the $X-r$ ay bursters and $\gamma-$ ray bursters, but I don't believe that they "fill in" to produce a continuum. Rather, they form a new "clump" that has little overlap with either X-ray bursters or $\gamma$-ray bursters.

S. Woosley: Besides its brilliance, the rapid rise time $(\sim 0.2 \mathrm{~ms})$ of the March 5 event is a hitherto unique characterisic of this particular gamma-ray burst. Does the class of $\mathrm{VS}^{2} \mathrm{~B}$ display this same rapid rise time?

D. Evans: Rise time data are not yet available from Signe and Prognoz and aren't determined by either the PVO or ISEE observations. This may well be the critical test of whether or not 790305 should be included as a VS ${ }^{2} \mathrm{~B}$.

S. Colgate: Is there any information concerning the intensity versus spectra on the $\mathrm{VS}^{2} \mathrm{Bs}$ ?

D. Evans: Not at this time. Neither PVO nor ISEE-C provide timeresolved data for these events. I believe that only Signe experiments or the Prognoz experiments might have these results later.

C. Alcock: Just how singular is 790305? If it had the same light curve, but was much fainter, would it be distinguishable from the rest of the $V S^{2} \mathrm{Bs}$ ?

D. Evans: The spectrum may be a bit harder than the average of soft bursts, but otherwise it would be very similar. 790305 had a very fast rise time ( $<1 / 4$ millisecond) and we don't yet know that parameter for the soft bursts. The extended emission from 790305 would not be observable if the overall strength were reduced by a factor of 100 to make it comparable to the soft bursts.

D. Helfand: If March 5 is the prototype of the $\mathrm{VS}^{2} \mathrm{Bs}$ then the obvious conclusion which I cannot resist stating is that this famous event had absolutely nothing to do with the LMC.

D. Evans: That is correct.

J. Grindlay: Your assertion that "traditional" gamma burst sources (with hard spectra, etc.) do not recur is in conflict with Schaeffer's optical results. How do you resolve this?

D. Evans: I actually made a rather different statement - i.e., that no recurrence from a "traditional" burst has been observed. It's possible to deduce from this that the recurrence time is probably greater than $\sim 10$ yrs. I believe that Brad Schaeffer concludes that he needs sources to repeat optically in 3-5 years to explain 
the optical flashes. I don't rememeber the uncertainty in this result. Maybe 10 yrs isn't inconsistent with his calculation. It's also possible tht optical flashes originate on $\gamma$-burst sources but are not always (or ever?) accompanied by $\gamma$-ray emission. 